\title{
7 Fazit und Ausblick
}

Der neu entwickelte hochdispersive Spiegel mit $-10000 \mathrm{fs}^{2}$ GDD konnte erfolgreich zum ersten $\mathrm{Mal}$ in einem femtosekunden Oszillator bei niedrigen Intrakavitätsleistungen eingesetzt werden. Es zeigten sich im Vergleich zu bereits bekannten Spiegelsätzen mit gleicher GDD im Rahmen der Genauigkeit keine Unterschiede. (Kapitel 3)

In einem nächsten Schritt muss die Leistungsskalierbarkeit untersucht werden, wobei besonderes Augenmerk auf das thermische Verhalten der Beschichtung und seine Zerstörschwelle gelegt werden muss.

Bei der Untersuchung des MAM zeigte sich, dass durch nichtlineare Effekte Störungen im stabilen CW-Resonator verstärkt werden. (Abschnitt 6.1) Es konnte auch bestätigt werden, dass die erwartete spektrale Verschiebung der Laseremission durch den MAM auftritt und durch den Einfallswinkel auf diesen variiert werden kann. (Abschnitt 6.2) Selbsterhaltende Modenkopplung durch die Nichtlinearität des MAM konnte nicht umgesetzt werden. Beim Anstieg der Spitzenintensität nach dem Start der ML konnte ein Temperaturanstieg auf der MAM-Oberfläche beobachtet werden (Abschnitt 6.3), welche nicht als Hauptursache der spektralen Verschiebung ausgeschlossen werden konnte.

Um den MAM erfolgreich als Modelocker einzusetzen, ist es notwendig noch zwei weitere Experimente durchzuführen. Zum einen muss die Auskoppelrate des MAM erhöht werden. Dies würde mehr Raum für ein stärkeres Ansteigen der Reflektivität lassen und damit die potentielle Modulationstiefe erhöhen. Hierzu wird ein Resonator mit höherem Verstärkungspotential benötigt, umgesetzt durch mehrere Reflexionen auf der TD [58], welcher sich im Moment im Aufbau befindet. Zum anderen muss untersucht werden, welchen Anteil thermische Effekte im Vergleich zu Nichtlinearitäten zu den Eigenschaften des MAM beitragen. Durch eine zeitaufgelöste Beobachtung des Spektrums könnte festgestellt werden, ob die Verschiebung instantan stattfindet oder nicht. 